\title{
Less hippocampal neuronal death in young gerbils following transient global cerebral ischemia is associated with long-term maintenance of insulin-like growth factor 1 and its receptors in the hippocampal CA1 region
}

\author{
BING CHUN YAN ${ }^{1-3 *}$, JIE WANG $^{1 *}$, JIANWEN CAO $^{1}$ and MOO-HO WON ${ }^{4}$ \\ ${ }^{1}$ Department of Traditional Chinese and Western Medicine,Jiangsu Key Laboratory of \\ Integrated Traditional Chinese and Western Medicine for Prevention and Treatment of Senile Diseases; \\ ${ }^{2}$ Department of Neurology, Affiliated Hospital of Yangzhou University, Yangzhou, Jiangsu 225001; \\ ${ }^{3}$ Jiangsu Key Laboratory of Zoonosis, Jiangsu Co-innovation Center for Prevention and Control of \\ Important Animal Infectious Diseases and Zoonoses, Yangzhou, Jiangsu 225009, P.R. China; ${ }^{4}$ Department of \\ Neurobiology, School of Medicine, Kangwon National University, Chuncheon, Gangwon 200-701, Republic of Korea
}

Received July 12, 2017; Accepted November 13, 2017

DOI: $10.3892 / \mathrm{mmr} .2017 .8243$

\begin{abstract}
Insulin-like growth factor 1 (IGF-1) is a well-known growth factor with well-defined neuroprotective effects against cerebral ischemia. However, the age-dependent differences in the expression of IGF-1 and its receptor (IGF-1R) in the brain following transient cerebral ischemia (TCI) have not been elucidated. In the present study, the differences in IGF-1 and IGF-1R in the gerbil hippocampal CA1 region of young and adult gerbils 5 min following TCI were determined. Seven days following TCI, the neuronal death in the hippocampal CA1 region of young gerbils was significantly less than that observed in adult gerbils. In addition, the immunoreactivity, and levels of IGF-1 and IGF-1R in the CA1 region of the normal young were higher than those in the normal adult. Four days following TCI, the immunoreactivity, and protein levels of IGF-1 and IGF-1R were markedly decreased in the adult group. By contrast, in the young group, the immunoreactivity and expression levels were much greater than those in the adult group. However, 7 days following TCI, all immunoreactivity and expression levels were markedly decreased when compared with those in the normal adult and young groups.
\end{abstract}

Correspondence to: Professor Bing Chun Yan, Department of Traditional Chinese and Western Medicine, Jiangsu Key Laboratory of Integrated Traditional Chinese and Western Medicine for Prevention and Treatment of Senile Diseases, 88 South University Avenue, Yangzhou, Jiangsu 225001, P.R. China

E-mail: bcyan@yzu.edu.cn

${ }^{*}$ Contributed equally

Key words: transient cerebral ischemia, insulin-like growth factor 1, delayed neuronal death, young animal
In addition, the immunoreactivity and expression levels in the young groups were significantly higher than those of the adult groups. In conclusion, the present study demonstrated that the higher and sustained expression of IGF-1 and IGF-1R in the young gerbil hippocampal CA1 region following TCI may be associated with the reduced neuronal death compared to that in the adults.

\section{Introduction}

Experimental transient cerebral ischemia (TCI) induced by the reduction of blood supply to the brain by the occlusion of the bilateral common carotid arteries results in irreversible neuronal damage in some sensitive brain regions, such as the hippocampus (1-3). The region I of hippocampus proper (CA1), in particular, is well known for the grading of the susceptibility to transient global cerebral ischemia $(4,5)$. The loss of neurons in the adult hippocampal CA1 region progressively occurs from day 3 to 4 after a transient ischemic injury, which is referred to as 'delayed neuronal death' (DND) (6). It is well known that the process of DND is different according to age. For instance, it has been reported that young animals are partly resistant to ischemia-induced neuronal damage (7). Also, our previous studies clearly showed that after $5 \mathrm{~min}$ of TCI DND occurred from day 7 to 10 in the young gerbil, which was considerably more delayed and less severe than that in the adult gerbils $(7,8)$.

The growth hormone insulin-like growth factor 1 (IGF-1) is a well-known growth factor with well-defined effects on many organs, including the brain (9). IGF-1 is highly expressed throughout the brain, including the cortex and hippocampus $(10,11)$. Many researchers have reported that IGF-1 participates in neuronal survival and glucose utilization in the hippocampus $(12,13)$. It is also reported that IGF-1 could regulate neuronal integration into the synaptic circuits of the hippocampus $(14,15)$. In addition, IGF-1 was found to promote 
synapse formation and prevent the death of neurons in neurodegenerative diseases $(15,16)$. Additionally, blunting IGF-1 was reported to cause an obvious decrease in the survival of neurons under basal conditions or after hypoxia/ischemia (17). Most of the IGF-1 actions are primarily mediated through its receptor (IGF-1R). Indeed, IGF-1 is the main ligand for IGF-1R (18), which is a multifunctional transmembrane tyrosine kinase $(19,20)$. In the nervous system, IGF-1 exerts its biological function by binding with IGF-1R, which can integrate with the enhancement of protein synthesis, cell survival and cell proliferation $(21,22)$. Recent research demonstrated that the IGF-1R also plays an essential role in neuroprotection following hypoxia/ischemia $(17,23)$.

The expression of IGF-1 and IGF-1R is significantly changed after cerebral ischemia injury (24). Also, some researchers have reported that IGF-1 and IGF-1R, which are expressed in the hippocampus, are associated with the loss of neurons $(25,26)$. In addition, it has also been reported that the expression levels of IGF-1 and IGF-1R decrease in an age-dependent manner (27). However, few studies have focused on the effect of the age-dependent change of IGF-1 and IGF-1R expression in the brain following TCI. Accordingly, in the present study, we examined the change in the expression of IGF-1 and IGF-1R in the hippocampal CA1 region of young gerbil (postnatal 1 month) after TCI and compared them with those in the adult gerbil (postnatal 6 month) after TCI (28).

\section{Materials and methods}

Experimental animals. The male Mongolian gerbils (Meriones unguiculatus) were bought from the Experimental Animal Center of the Kangwon National University (Chuncheon, South Korea). Mongolian gerbils aged 1-month old (body weight, 25-30 g) were divided into the young group and those aged 6 months old (body weight, 65-75 g) were divided into the adult group. The animals were housed in pathogen-free conditions at $23 \pm 2^{\circ} \mathrm{C}$ and $58 \pm 2 \%$ relative humidity, with a 12 -h light $/ 12-\mathrm{h}$ dark cycle, and had free access to food and water. Procedures involving animals handling and their care were conformed to current international laws and policies (Guide for the Care and Use of Laboratory Animals, the National Academies Press, 8th Edition, 2011). All efforts were made to minimize animal suffering and the number of experimental animals. The animal protocol was approved based on ethical procedures and scientific care by the Yangzhou University-Institutional Animal Care and Use Committee (YIACUC-14-0013).

Induction of TCI. Transient global cerebral ischemia was induced as follows, which was described previously (29). The bilateral common carotid artery of gerbil was occluded using non-traumatic aneurysm clips under anesthesia by inhalation of $2.5 \%$ isoflurane in $33 \%$ oxygen and $67 \%$ nitrous oxide. The complete interruption of blood flow was confirmed by observing the central artery in retinae using an ophthalmoscope. After 5 min of occlusion, the aneurysm clips were removed from the common carotid arteries. The restoration of blood flow (reperfusion) was observed directly using the ophthalmoscope. The body (rectal) temperature under free-regulating or normothermic $\left(37 \pm 0.5^{\circ} \mathrm{C}\right)$ conditions was monitored with a rectal temperature probe. Meanwhile, the body temperature was maintained by a thermometric blanket before, during and after the surgery until the animals completely recovered from anesthesia. Thereafter, animals were kept on the thermal incubator to maintain the body temperature until the animals were euthanized. Sham-operated animals were subjected to the same surgical procedures except that the common carotid arteries were not occluded.

Tissue processing for histology. Tissues were collected as previously described (30), sham-( $\mathrm{n}=7$ at each time point) and ischemia-operated young ( $\mathrm{n}=7$ at each time point) and adult ( $n=7$ at each time point) gerbils at designated times (1, 4 and 7 days after reperfusion) were sacrificed. The animals were anesthetized with pentobarbital sodium and perfused transcardially with $0.1 \mathrm{M}$ phosphate-buffered saline (PBS, $\mathrm{pH} 7.4$ ) followed by $4 \%$ paraformaldehyde in $0.1 \mathrm{M}$ phosphate-buffer (PB, pH 7.4). The brains were removed and postfixed in the same fixative for $6 \mathrm{~h}$. The brain tissues were cryoprotected by infiltration with $30 \%$ sucrose overnight. Thereafter, frozen tissues were serially sectioned on a cryostat (Leica Microsystems GmbH, Wetzlar, Germany) into 30- $\mu$ m coronal sections, and they were then collected into 6-well plates containing PBS.

Immunohistochemistry. Immunohistochemistry was performed according to a previously published procedure (30). To examine the change of Neuronal nuclei (NeuN), IGF-1 and its receptors in the CA1 after ischemia-reperfusion, we carried out immunohistochemical staining with rabbit anti-NEUN (1:1,000; Cell Signaling Technology, Inc., Danvers, MA, USA), rabbit anti-IGF-1 and IGF-1R (1:200; Santa Cruz Biotechnology, Inc., Dallas, TX, USA), and biotinylated goat anti-rabbit IgG (1:250; Vector Laboratories, Inc., Burlingame, CA, USA) for secondary antibody. In order to establish the specificity of the immunostaining, a negative control test was carried out with pre-immune serum instead of primary antibody. The negative control resulted in the absence of immunoreactivity in all structures.

Densities of NEUN, IGF-1 and IGF-1R immunoreactive structures were measured as previously described (11), Digital images of the hippocampal subregions were captured with an image analyzing system equipped with a computer-based microscope (Nikon Corporation, Tokyo, Japan). Cell counts were obtained by averaging the counts from the sections taken from each animal. In addition, the staining intensity of IGF-1 and IGF-1R immunoreactive structures was evaluated on the basis of an optical density (OD), which was obtained after the transformation of the mean gray level using the formula: OD=log (256/mean gray level). The OD of background was taken from areas adjacent to the measured area. After the background density was subtracted, a ratio of the OD of image file was calibrated as \% (relative OD, ROD) using Adobe Photoshop version 8.0 and then analyzed using NIH Image 1.59 software. All measurements were performed in order to ensure objectivity in blind conditions, by two observers for each experiment, carrying out the measures of experimental samples under the same conditions.

Western blot analysis. In order to examine the protein levels of IGF-1 and its receptors in the ischemic CA1 region, the 
animals ( $n=7$ at each time point) were used for western blot analysis sham, 4, 7 days after the ischemic surgery in the young and adult group. Western blot analysis was performed out according to a previously published procedure (31). After sacrificing them and removing the brains, they were serially and transversely cut into a thickness of $400 \mu \mathrm{m}$ on a vibratome, and the hippocampus was dissected with a surgical blade. They were preprocessed by Whole Cell Lysis Assay kit (KeyGEN, Nanjing, China)/Total Protein Extraction kit. Protein concentrations were determined using a Pierce BCA Protein Assay kit (Thermo Fisher Scientific, Inc., Waltham, MA, USA). Equal amounts of protein $(30 \mu \mathrm{g})$ were separated by $10 \%$ sodium dodecyl sulphate-polyacrylamide gel electrophoresis (SDS-PAGE) and transferred to nitrocellulose membranes (EMD Millipore, Bedford, MA, USA). In order to incubate antibodies, the same nitrocellulose membranes striped were used. To reduce background staining, the membranes were incubated with 5\% BSA in TBS containing 0.1\% Tween-20 for $60 \mathrm{~min}$, followed by incubation with rabbit anti-IGF-1, IGF-1R (1:1,000; Santa Cruz Biotechnology, Inc.) and $\beta$-actin (1:1,000; Cell Signaling Technology, Inc.) overnight at $4^{\circ} \mathrm{C}$ and subsequently exposed to secondary goat anti-rabbit $\mathrm{IgG}$ (Santa Cruz Biotechnology, Inc.) for $2 \mathrm{~h}$ at room temperature and the SuperSignal West Pico Chemiluminescent Substrate (Thermo Fisher Scientific, Inc.) was used for protein detection. The result of western blot analysis was scanned, and densitometric analysis for the quantification of the bands was done using Quantity One Analysis Software (Bio-Rad Laboratories, Inc., Hercules, CA, USA), which was used to count relative optical density (ROD): A ratio of the ROD was calibrated as \%, with sham-group designated as $100 \%$. Each blot shown is representative of at least three similar independent experiments.

Reverse transcription-quantitative polymerase chain reaction (RT-qPCR). Total RNA was extracted from hippocampus of brain tissues using TRIzol reagent (Invitrogen; Thermo Fisher Scientific, Inc.) according to the manufacturer's instructions. Then, total RNA ( $2 \mu \mathrm{g}$ ) was purified with the purification kit (Thermo Fisher Scientific, Inc.), and reversely transcribed into cDNA using the PrimeScript ${ }^{\mathrm{TM}}$ II 1st Strand cDNA synthesis kit (Takara Bio, Inc., Dalian, Japan) according to the manufacturer's instructions. RT-qPCR was performed using the PrimeScript ${ }^{\mathrm{TM}}$ RT Master Mix (Takara Bio, Inc.) on a LightCycler ${ }^{\circledR} 96$ Real-Time PCR System (Roche Diagnostics, Basel, Switzerland). The 2-step PCR was performed: $95^{\circ} \mathrm{C}$ for $10 \mathrm{sec}$ and 40 cycles of $95^{\circ} \mathrm{C}$ for $5 \mathrm{sec}$ and $60^{\circ} \mathrm{C}$ for $30 \mathrm{sec}$. The RNA U6 mRNA was used as internal controls. All the reactions were conducted in triplicate. Primers for U6 were: 5'-GGAACGATACAGAGAAGATTAGC-3' (forward) and 5'-TGGAACGCTTCACGAATTTGCG-3' (reverse). Primers for IGF-1 were 5'-CTGGACCAGAGACCCTTT GC-3' (forward) and 5'-GGACGGGGACTTCTGAGTCTT-3' (reverse); Primers for IGF-1R were 5'-ACACGCAACGAG ACTAATCG-3' (forward) and 5'-TTAGAGACTGAGCGG CATCC-3' (reverse). Data are shown as the fold-change.

Statistical analysis. Data are expressed as the mean \pm SEM. The data were evaluated by a one-way ANOVA SPSS program, and the means assessed using Duncan's multiple-range test.

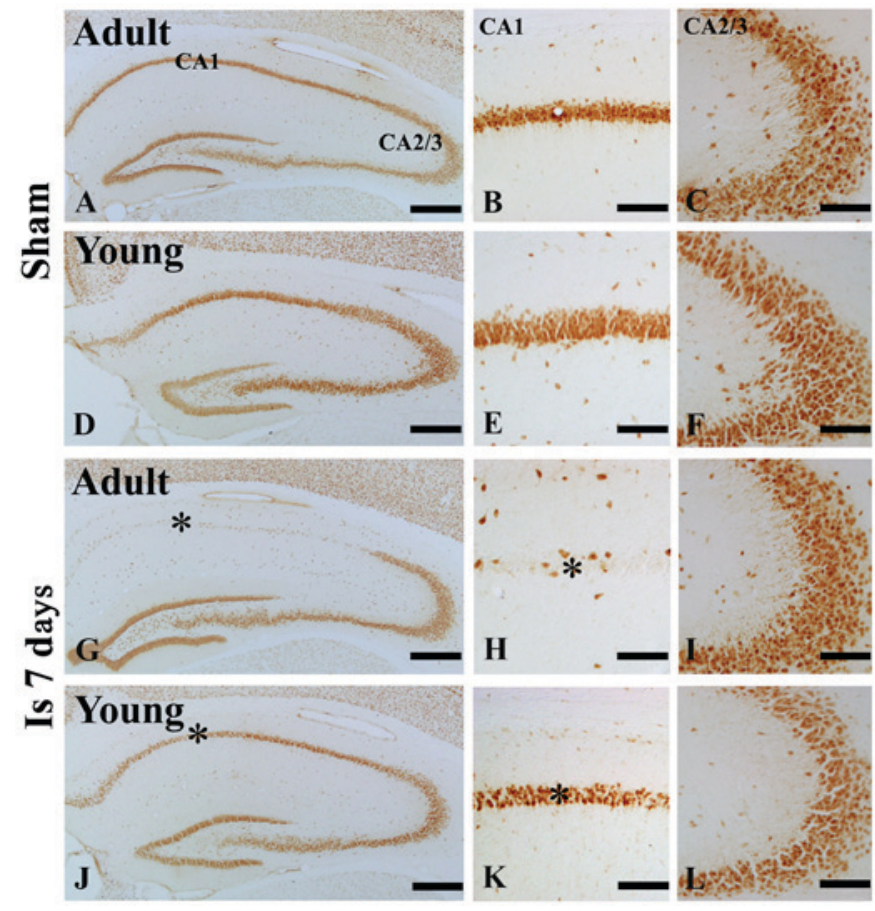

Figure 1. NeuN immunohistochemistry in the hippocampal CA1 and CA 2/3 region of the (A-F) sham and (G-L) 7 day post-ischemia groups in the adultand young gerbils. The number of NeuN immunoreactive cells in the 7 day post ischemia group of young gerbils was greater than that observed in the adults. Scale bars $=200 \mu \mathrm{m}$ (A, D, G and J) and $50 \mu \mathrm{m}$ (B, C, E, F, H, I, K and L). The * on each image indicates the expression of NeuN. NeuN, neuronal nuclei; CA, cornus ammonis; Is $7 \mathrm{~d}, 7$ day post-ischemia group.

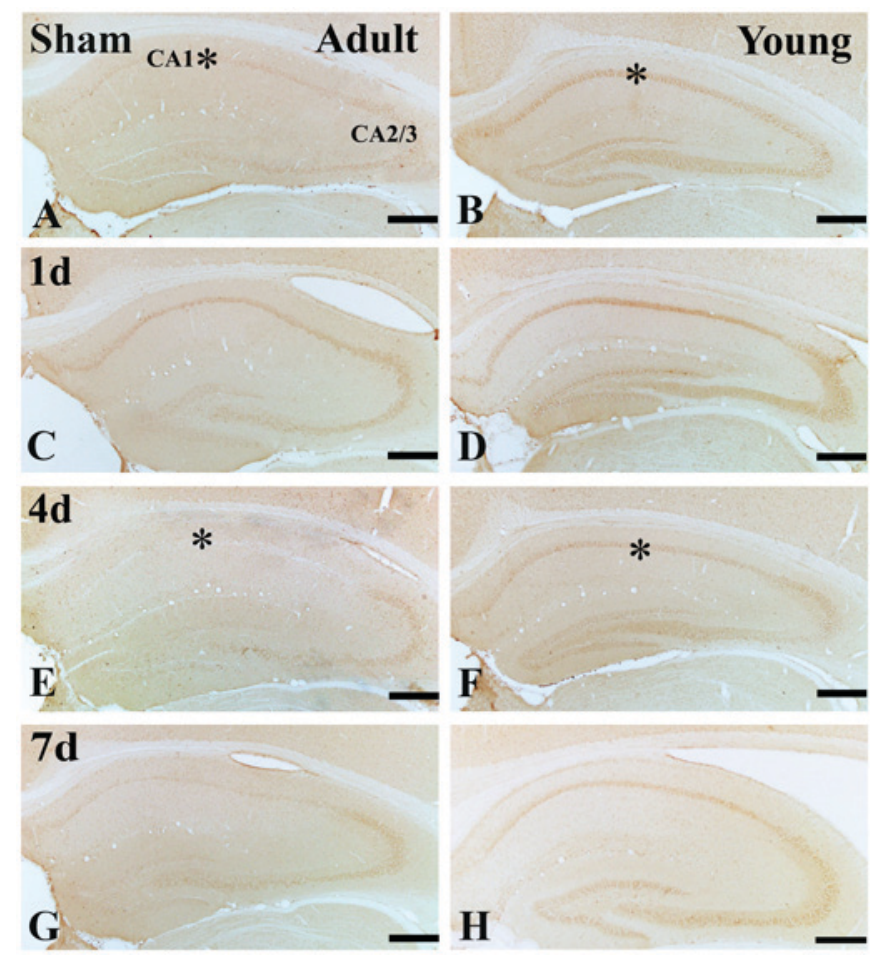

Figure 2. Immunohistochemistry for IGF-1 in the adult and young (A and B) sham- and the ischemia groups: (C and D) 1 day, (E and F) 4 days and ( $\mathrm{G}$ and $\mathrm{H}$ ) 7 days. IGF-1 immunoreactivity in the SP of the young sham-group is higher than that in the adult sham-group. At 4 days post-ischemia, IGF-1 immunoreactivity in the adult SP is distinctively decreased; however, at this time, IGF-1 immunoreactivity in the young SP is sustained. Scale bar $=200 \mu \mathrm{m}$. The * on each image indicates the expression of IGF-1 or the IGF-1 receptor in the SP. SP, stratum pyramidale; IGF-1, insulin-like growth factor 1. 

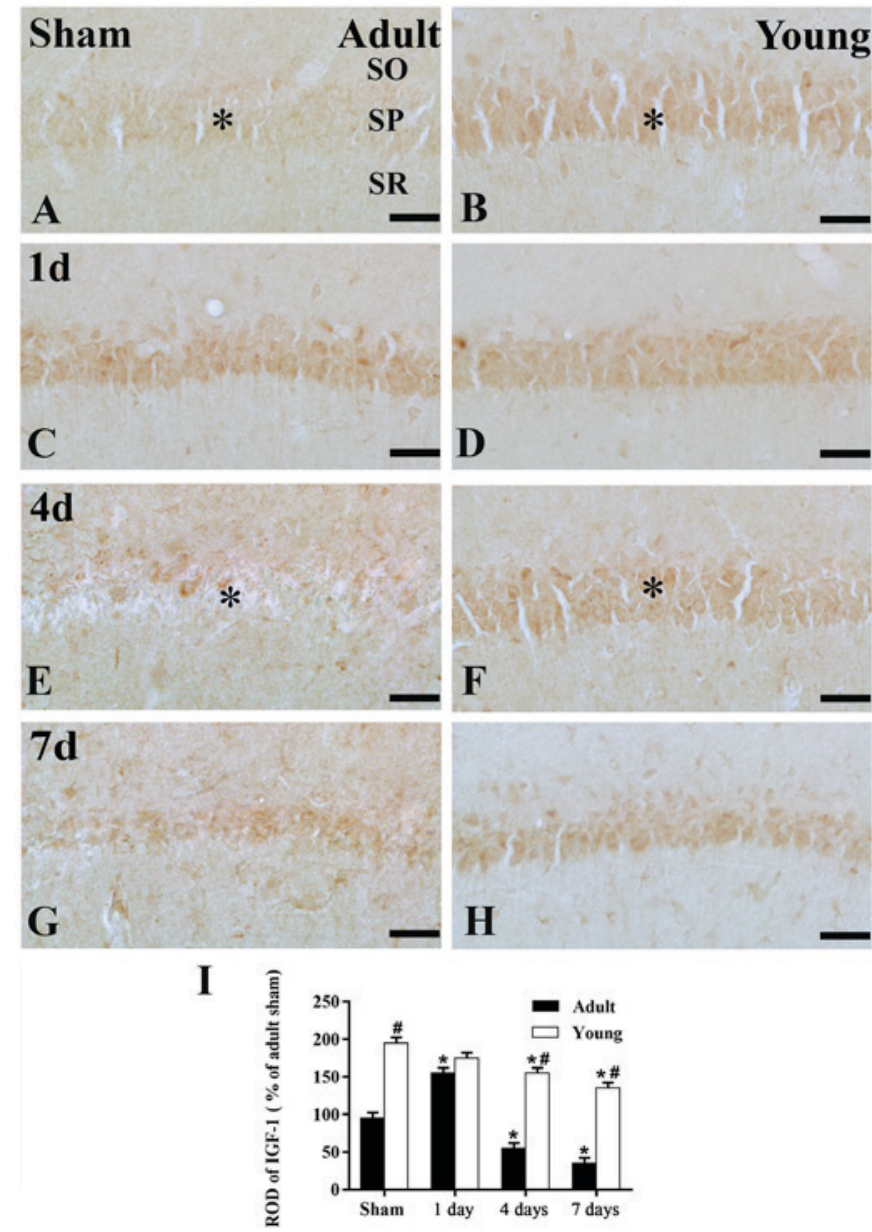

Figure 3. Immunohistochemistry for IGF-1 in the adult and young of the (A and B) sham- and the ischemia groups: (C and D) 1 day, (E and F) 4 days and $(\mathrm{G}$ and $\mathrm{H}) 7$ days. Scale bar=50 $\mu \mathrm{m}$. (I) ROD as a percentage of the IGF-1 immunoreactivity. Data are presented as the mean \pm standard error of the mean ( $\mathrm{n}=7$ per group). The ${ }^{*}$ on each image indicates the expression of IGF-1 or the IGF-1 receptor in the SP. ${ }^{*} \mathrm{P}<0.05$ vs. the sham group; ${ }^{*} \mathrm{P}<0.05$, vs. the respectively preceding group. IGF-1, insulin-like growth factor $1 ; \mathrm{SO}$, stratum oriens; SP, stratum pyramidale; SR, stratum radiatum; ROD, relative optical density.

$\mathrm{P}<0.05$ was considered to indicate a statistically significant difference.

\section{Results}

Neuronal damages. In the adult sham-group, NeuN immunoreactive neurons were easily observed in the striatum pyramidale (SP) of the hippocampus including CA1-3 region (Fig. 1A-C). However, seven day after ischemia/reperfusion (I/R), a few NeuN immunoreactive neurons were found in the SP of the hippocampal CA1 not CA2-3 region (Fig. 1G-I).

In the young sham-group, NeuN immunoreactive neurons in the SP of the hippocampus were also well observed (Fig. 1D-F). In the 7 days after I/R, the number of NeuN immunoreactive neurons in the SP was significantly much more than that in the adult group (Fig. 1J-L).

IGF-1 immunoreactivity. In the adult sham-group, IGF-1 immunoreactivity in the SP was much lower than that of the young sham-group (Figs. 2A and B; and3A, B and I). One day
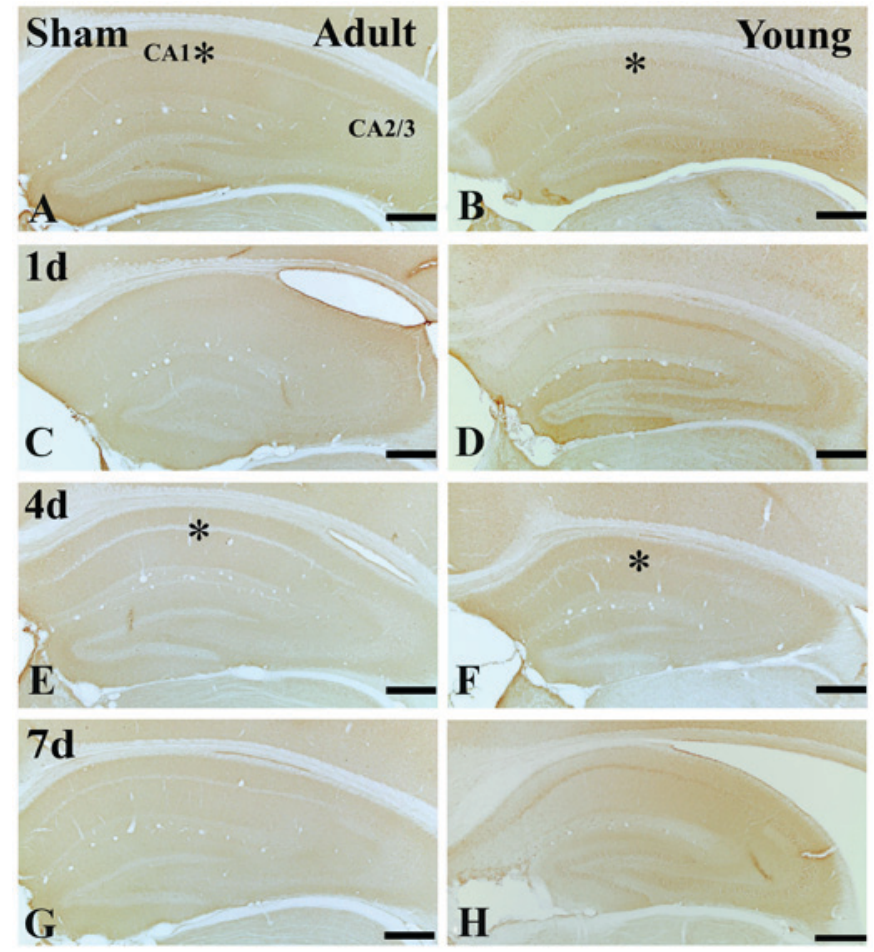

Figure 4. IGF-1R immunohistochemistry in the adult and young of the (A and B) sham- and the ischemia groups: (C and D) 1 day, ( $E$ and F) 4 days and $(\mathrm{G}$ and $\mathrm{H}) 7$ days. IGF-1R immunoreactivity in the SP of the adult group is markedly decreased 4 days following ischemia-reperfusion. In the young groups, IGF-1R immunoreactivity in the SP is well maintained until 4 days following ischemia-reperfusion. Scale bar $=200 \mu \mathrm{m}$. The ${ }^{*}$ on each image indicates the expression of IGF-1 or the IGF-1 receptor in the SP. IGF-1R, insulin-like growth factor 1 receptor; SP, stratum pyramidale.

after I/R, IGF-1 immunoreactivity in the SP was increased (Figs. 2C; and 3C and I), its immunoreactivity was apparently decreased at 4 days after I/R (Figs. 2E; and 3E and I). Seven days after $\mathrm{I} / \mathrm{R}$, the immunoreactivity was similar to that at 4 days after I/R. (Figs. 2B; and 3B and I).

In the young sham-group, strong IGF-1 immunoreactivity was found in the SP of the CA1 region (Figs. 2B and 3B). IGF-1 immunoreactivity was maintained until 4 days post-ischemia in the SP of the CA1 region (Figs. 2D and F; and 3D and F). Seven days after I/R, IGF-1 immunoreactivity in the SP was obviously decreased (Figs. 2H; and $3 \mathrm{H}$ and I).

IGF-1R immunoreactivity. In the adult sham-group, IGF-1R immunoreactivity in the CA1 region was much lower than that in the young sham-group (Figs. 4A, B and E; and 5A, B and E). One day after I/R, IGF-1R immunoreactivity was decreased in SP (Figs. 4C; and 5C and I). Four days after I/R, IGF-1R immunoreactivity was hardly detected in SP of the CA1 region (Figs. 4E and 5E). Thereafter, IGF-1R immunoreactivity in the SP was weakly detected in the CA1 region (Figs. $4 \mathrm{G}$ and $5 \mathrm{G}$ ).

In the young sham-group, IGF-1R immunoreactivity in the CA1 region was strongly detected in the SP of CA1 region. (Figs. 4B and 5B). All groups after I/R, IGF-1R immunoreactivity in the SP of young was much higher than that in the adult (Figs. 4D, F and H; and 5F, H and I).

Changes in $m R N A$ and protein levels of IGF-1/IGF-1R. The mRNA and protein levels of IGF-1 and IGF-1R were much 


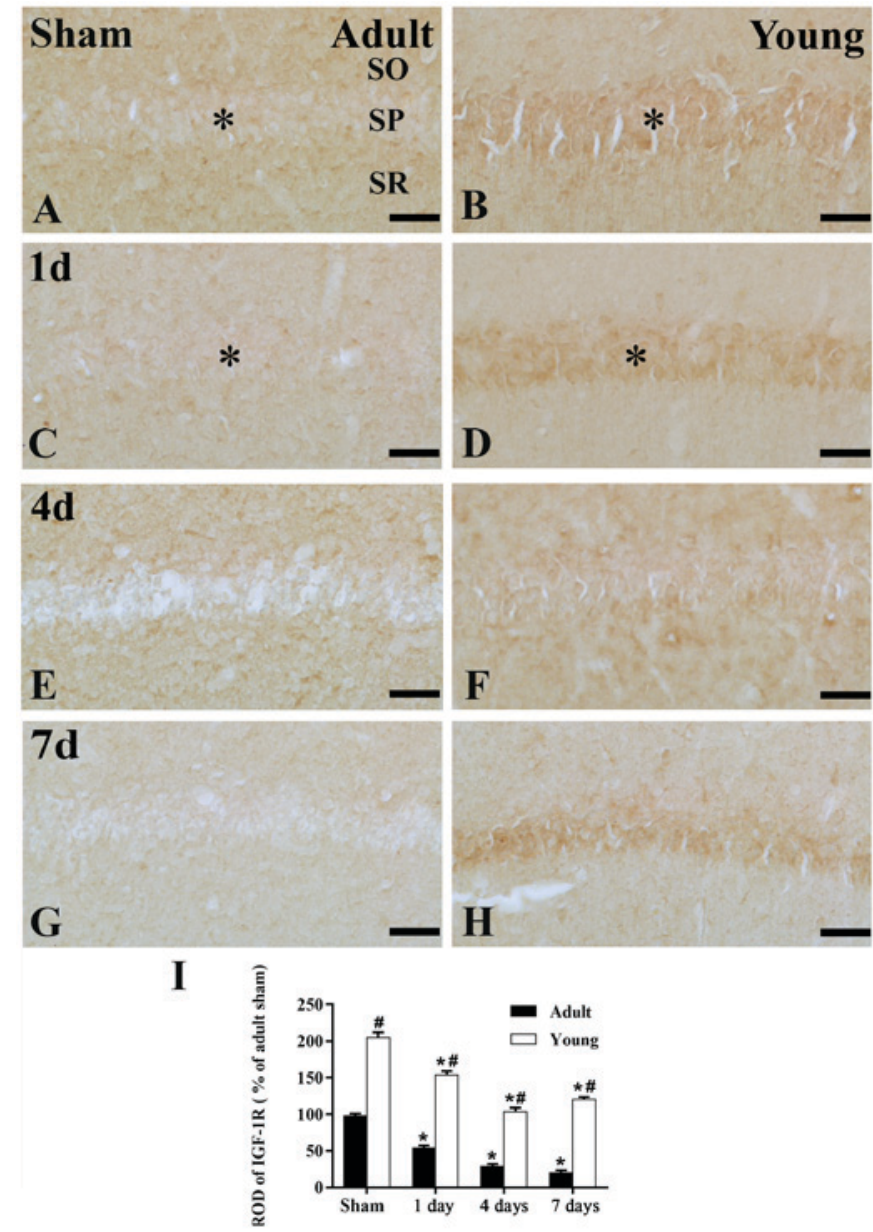

Figure 5. IGF-1R immunohistochemistry in the adult and young of the (A and B) sham- and the ischemia groups: (C and D) 1 day, $(\mathrm{E}$ and $\mathrm{F}) 4$ days and (G and $\mathrm{H}) 7$ days. (I) ROD as a percentage of the IGF-1R. Data are presented as the mean \pm standard error of the mean ( $\mathrm{n}=7$ per group). ${ }^{*} \mathrm{P}<0.05$ vs. the sham group; ${ }^{\#} \mathrm{P}<0.05$ vs. the corresponding adult group. Scale bar $=50 \mu \mathrm{m}$. The * on each image indicates the expression of IGF-1 or the IGF-1 receptor in the SP. IGF-1R, insulin-like growth factor 1 receptor; SO, stratum oriens; SP, stratum pyramidale; SR, stratum radiatum; ROD, relative optical density.

higher in the young sham-group than those in the adult sham-group (Fig. 6). Four and seven days after I/R, the mRNA and protein levels of IGF-1 and IGF-1R were particularly decreased in the adult group. However, in the young group at this time after I/R, They were significantly higher than those in the corresponding adult-group (Fig. 6A-E).

\section{Discussion}

Among the multiple risk factors for ischemic stroke, age plays a key role in the brain injury induced by cerebral ischemic stroke (8). Our previous studies have demonstrated that young gerbils have a stronger to anti-cerebral ischemia activity than the adult gerbils under the same condition $(7,32)$. In addition, it has been reported that in the young gerbil neuronal death occurs in the hippocampal CA1 region 7 days after TCI, which is later than it occurs in the adult gerbil (33).

In this study, we found that the immunoreactivity and their RNA and protein expression levels of IGF-1 and IGF-1R in the young gerbil were much higher than those in the adult gerbil
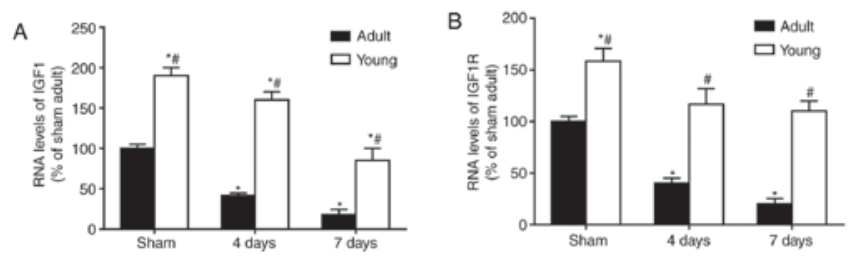

C
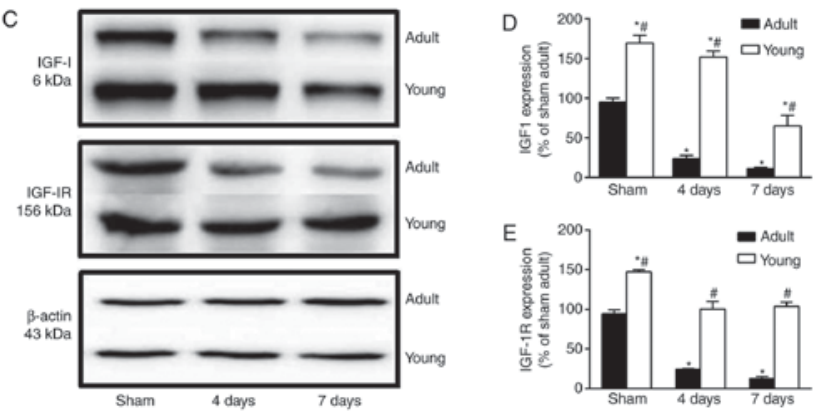

Figure 6. Western blot analysis and reverse transcription-quantitative polymerase chain reaction of IGF-1 and IGF-1R in the CA1 of the young and adult groups ( $n=7 /$ group) following ischemia-reperfusion. The RNA levels of (A) IGF-1 and (B) IGF-1R in the hippocampal CA1 region of gerbils. (C) The protein levels of (D) IGF-1 and (E) IGF-1R in the in the hippocampal CA1 region of gerbils. ROD as a percentage of the immunoblot band is presented. Data are presented as the mean \pm standard deviation ( $\mathrm{n}=7$ /group) ${ }^{*} \mathrm{P}<0.05$ vs. the sham group; ${ }^{\#} \mathrm{P}<0.05$, vs. the corresponding adult group. IGF-1, insulin-like growth factor 1; IGF-1R, insulin-like growth factor 1 receptor; ROD, relative optical density.

under physiological conditions. This finding is consistent with a previous study that showed that the immunoreactivity and protein levels of IGF-1 and IGF-1R were significantly decreased with age (27). Many researchers have reported that IGF-1 and IGF-1R act as the key modulator of brain glucose to participate in neuroprotection $(12,34)$. Glucose is the major source of metabolic energy for the central nervous system (CNS), thus it is very important to provide sufficient glucose to the brain (35). It is well known that impairment of the energy metabolism in the brain is one of the important mechanisms of cerebral ischemic injury (36). GLUT-1, the predominant glucose transporter specifically expressed by capillary endothelial cells of the brain, is of great importance in regulating the transportation and level of glucose in the brain, and its expression reflects the rate of cerebral glucose utilization $(1,37,38)$. Park et al reported that the higher expression of GLUT-1in the hippocampal CA1 region of the young gerbils after TCI may contribute to less and more delayed neuronal death in the young gerbil (1). Some studies have reported that IGF-1 and IGF-1R contributed to increase glucose metabolism, which indicated that the elevated expression of IGF-1 and IGF-1R expression was associated with neuroprotection after TCI $(21,39)$.

We additionally compared changes of IGF-1and IGF-1R in the CA1 region between adult and young gerbils after ischemia-reperfusion. The IGF-1 immunoreactivity and its protein level in the CA1 region of adult hippocampus were increase at earlier time and then dramatically decreased. Hwang et al have demonstrated that the expression of IGF-1 was transiently increased in the hippocampus and cerebral cortex after I/R injury, which may be associated with the short resistance to DND after ischemic insult (40). However, in the young gerbil after ischemia-reperfusion, the immunoreactivity and mRNA and protein expression levels of IGF-1 was sustained 
until day 4 after ischemia-reperfusion in the hippocampal CA1 region. Certain researchers reported that endogenous IGF-1 and IGF-1R were involved in the neuroprotective effect against ischemic damage in the brain (41-44). Activation of IGF-1/IGF-1R stimulates the PI-3K/Akt pathway and inhibits the GSK-3 $\beta$ pathway, to exert their effect on the antioxidant defense of neuron-, metabolism of glucose- and synthesis of anti-apoptotic-associated proteins, which result in the protective effect and ultimately in neuronal survival $(21,45)$. It is particularly noteworthy that the Akt signaling pathway, as an important upstream signaling pathway, plays an important role in the survival and repair of neuronal cells after cerebral ischemia $(46,47)$. The activation of Akt can control multiple intracellular signals, such as the mTOR signaling pathway, GSK-3 $\beta$ signaling pathway etc. Then, the downstream signaling pathways can promote proliferation and survival after cerebral ischemia (48-50). In addition, some studies reported that treatment with IGF-1 after an ischemic stroke partially improved the ischemic damage of neurons induced by ischemia-reperfusion injury $(51,52)$. Therefore, the reduced neuronal death in the hippocampal CA1 region of the young gerbils after TCI compared to that in the adults may be associated with the higher and sustained expression of IGF-1 and IGF-1R. Furthermore, the relevant molecular biological mechanisms may be associated with the Akt signaling pathway.

In conclusion, our present findings indicated that the expression levels of IGF-1 and IGF-1R in the hippocampal CA1 region in the normal young gerbils were much higher than those in the normal adult. Additionally, their sustained expression levels in the hippocampal CA1 region after ischemia-reperfusion may serve as the evidence to explain the reason for the more delayed and reduced neuronal death/damage in the young gerbil. Also, it could be hypothesized that increasing the levels of IGF-1/IGF-1R has potential as an alternative target for the prevention of ischemic damage in the brain.

\section{Acknowledgements}

This study was supported by the National Natural Science Foundation of China (no. 81401005), The Natural Science Foundation of Jiangsu Province of China (no. BK20140494), Key University Science Research Project of Jiangsu Province (no. 16KJA310006), China Postdoctoral Science Foundation (no. 2014M561720) and Postdoctoral Science Foundation of Jiangsu Province (no. 1401155C).

\section{References}

1. Park SM, Lee JC, Chen BH, Shin BN, Cho JH, Kim IH, Park JH, Won MH, Ahn JH, Tae HJ, et al: Difference in transient ischemia-induced neuronal damage and glucose transporter-1 immunoreactivity in the hippocampus between adult and young gerbils. Iran J Basic Med Sci 19: 521-528, 2016.

2. Bae EJ, Chen BH, Yan BC, Shin BN, Cho JH, Kim IH, Ahn JH, Lee JC, Tae HJ, Hong S, et al: Delayed hippocampal neuronal death in young gerbil following transient global cerebral ischemia is related to higher and longer-term expression of p63 in the ischemic hippocampus. Neural Regen Res 10: 944-950, 2015.

3. Lee JC, Tae HJ, Chen BH, Cho JH, Kim IH, Ahn JH, Park JH, Shin BN, Lee HY, Cho YS, et al: Failure in neuroprotection of remote limb ischemic postconditioning in the hippocampus of a gerbil model of transient cerebral ischemia. J Neurol Sci 358: $377-384,2015$.
4. Araki T, Kato H and Kogure K: Selective neuronal vulnerability following transient cerebral ischemia in the gerbil: Distribution and time course. Acta Neurol Scand 80: 548-553, 1989.

5. Domoráková I, Burda J, Mechírová E and Feriková M: Mapping of rat hippocampal neurons with NeuN after ischemia/reperfusion and Ginkgo biloba extract (EGb 761) pretreatment. Cell Mol Neurobiol 26: 1193-1204, 2006.

6. Kirino T: Delayed neuronal death in the gerbil hippocampus following ischemia. Brain Res 239: 57-69, 1982.

7. Oguro K, Miyawaki T, Yokota H, Kato K, Kamiya T, Katayama Y, Fukaya M, Watanabe M and Shimazaki K: Upregulation of GluR2 decreases intracellular Ca2+ following ischemia in developing gerbils. Neurosci Lett 364: 101-105, 2004.

8. Yan BC, Park JH, Ahn JH, Lee YJ, Lee TH, Lee CH, Cho JH, Kim MJ, Kim TY, Kang IJ and Won MH: Comparison of the immunoreactivity of Trx $2 / \operatorname{Prx} 3$ redox system in the hippocampal CA1 region between the young and adult gerbil induced by transient cerebral ischemia. Neurochem Res 37: 1019-1030, 2012.

9. Kartal Ö, Aydınöz S, Kartal AT, Kelestemur T, Caglayan AB Beker MC, Karademir F, Süleymanoğlu S, Kul M, Yulug B and Kilic E: Time dependent impact of perinatal hypoxia on growth hormone, insulin-like growth factor 1 and insulin-like growth factor binding protein-3. Metab Brain Dis 31: 827-835, 2016.

10. Tarantini S, Tucsek Z, Valcarcel-Ares MN, Toth P, Gautam T, Giles CB, Ballabh P, Wei JY, Wren JD, Ashpole NM, et al: Circulating IGF-1 deficiency exacerbates hypertension-induced microvascular rarefaction in the mouse hippocampus and retrosplenial cortex: Implications for cerebromicrovascular and brain aging. Age (Dordr) 38: 273-289, 2016.

11. Lee CH, Ahn JH, Park JH, Yan BC, Kim IH, Lee DH, Cho JH, Chen BH, Lee JC, Cho JH, et al: Decreased insulin-like growth factor-I and its receptor expression in the hippocampus and somatosensory cortex of the aged mouse. Neurochem Res 39: 770-776, 2014.

12. Bondy CA and Cheng CM: Insulin-like growth factor-1 promotes neuronal glucose utilization during brain development and repair processes. Int Rev Neurobiol 51: 189-217, 2002.

13. Cheng CM, Cohen M, Tseng V and Bondy CA: Endogenous IGF1 enhances cell survival in the postnatal dentate gyrus. J Neurosci Res 64: 341-347, 2001.

14. Nieto-Estévez V, Defterali Ç and Vicario-Abejón C: IGF-I: A key growth factor that regulates neurogenesis and synaptogenesis from embryonic to adult stages of the brain. Front Neurosci 10: 52, 2016.

15. Liu W, Ye P, O'Kusky JR and D'Ercole AJ: Type 1 insulin-like growth factor receptor signaling is essential for the development of the hippocampal formation and dentate gyrus. J Neurosci Res 87: 2821-2832, 2009.

16. Carro E, Trejo JL, Gomez-Isla T, LeRoith D and Torres-Aleman I: Serum insulin-like growth factor I regulates brain amyloid-beta levels. Nat Med 8: 1390-1397, 2002.

17. Liu W, D'Ercole JA and Ye P: Blunting type 1 insulin-like growth factor receptor expression exacerbates neuronal apoptosis following hypoxic/ischemic injury. BMC Neurosci 12: 64, 2011.

18. LeRoith D, Werner H, Beitner-Johnson D and Roberts CT Jr: Molecular and cellular aspects of the insulin-like growth factor I receptor. Endocr Rev 16: 143-163, 1995.

19. Czech MP: Signal transmission by the insulin-like growth factors. Cell 59: 235-238, 1989.

20. Gazit N, Vertkin I, Shapira I, Helm M, Slomowitz E, Sheiba M, Mor Y, Rizzoli S and Slutsky I: IGF-1 receptor differentially regulates spontaneous and evoked transmission via mitochondria at hippocampal synapses. Neuron 89: 583-597, 2016.

21. Cheng CM, Reinhardt RR, Lee WH, Joncas G, Patel SC and Bondy CA: Insulin-like growth factor 1 regulates developing brain glucose metabolism. Proc Natl Acad Sci USA 97: 10236-10241, 2000.

22. Madathil SK and Saatman KE: IGF-1/IGF-R Signaling in Traumatic Brain Injury: Impact on cell survival, neurogenesis and behavioral outcome. In: Brain Neurotrauma: Molecular, Neuropsychological and Rehabilitation Aspects. Kobeissy FH (ed). CRC Press/Taylor \& Francis, Boca Raton, FL, 2015.

23. Gualco E, Wang JY, Del Valle L, Urbanska K, Peruzzi F, Khalili K, Amini S and Reiss K: IGF-IR in neuroprotection and brain tumors. Front Biosci 14: 352-375, 2009.

24. Song Y, Pimentel C, Walters K, Boller L, Ghiasvand S, Liu J, Staley KJ and Berdichevsky Y: Neuroprotective levels of IGF-1 exacerbate epileptogenesis after brain injury. Sci Rep 6: 32095, 2016. 
25. Yang Y, Li X, Sun Q, He B, Jia Y, Cai D and Zhao R: Folate deprivation induces cell cycle arrest at G0/G1 phase and apoptosis in hippocampal neuron cells through down-regulation of IGF-1 signaling pathway. Int J Biochem Cell Biol 79: 222-230, 2016.

26. Werner $H$ and LeRoith $D$ : Insulin and insulin-like growth factor receptors in the brain: Physiological and pathological aspects. Eur Neuropsychopharmacol 24: 1947-1953, 2014.

27. Sonntag WE, Lynch CD, Bennett SA, Khan AS, Thornton PL, Cooney PT, Ingram RL, McShane T and Brunso-Bechtold JK: Alterations in insulin-like growth factor-1 gene and protein expression and type 1 insulin-like growth factor receptors in the brains of ageing rats. Neuroscience 88: 269-279, 1999.

28. Pentón-Rol G, Marin-Prida J, Pardo-Andreu G, Martínez-Sánchez G, Acosta-Medina EF, Valdivia-Acosta A, Lagumersindez-Denis N, Rodríguez-Jiménez E, Llópiz-Arzuaga A, López-Saura PA, et al: C-Phycocyanin is neuroprotective against global cerebral ischemia/reperfusion injury in gerbils. Brain Res Bull 86: 42-52, 2011.

29. Yan BC, Park JH, Ahn JH, Kim IH, Lee JC, Yoo KY, Choi JH, Hwang IK, Cho JH, Kwon YG, et al: Effects of high-fat diet on neuronal damage, gliosis, inflammatory process and oxidative stress in the hippocampus induced by transient cerebral ischemia. Neurochem Res 39: 2465-2478, 2014.

30. Yan BC, Ohk TG, Ahn JH, Park JH, Chen BH, Lee JC, Lee CH, Shin MC, Hwang IK, Moon SM, et al: Differences in neuronal damage and gliosis in the hippocampus between young and adult gerbils induced by long duration of transient cerebral ischemia. J Neurol Sci 337: 129-136, 2014

31. Shen H, Wang J, Jiang D, Xu P, Zhu X, Zhang Y, Yu X, Won MH, Su PQ and Yan BC: Topiramate improves neuroblast differentiation of hippocampal dentate gyrus in the D-galactose-induced aging mice via its antioxidant effects. Cell Mol Neurobiol 37: 869-877, 2017.

32. Kusumoto M, Arai H, Mori K and Sato K: Resistance to cerebral ischemia in developing gerbils. J Cereb Blood Flow Metab 15 886-891, 1995.

33. Yan BC, Park JH, Lee CH, Yoo KY, Choi JH, Lee YJ, Cho JH, Baek YY, Kim YM and Won MH: Increases of antioxidants are related to more delayed neuronal death in the hippocampal CA1 region of the young gerbil induced by transient cerebral ischemia. Brain Res 1425: 142-154, 2011

34. Hernandez-Garzón E, Fernandez AM, Perez-Alvarez A, Genis L, Bascuñana P,Fernandez de la Rosa R, Delgado M, Angel Pozo M, Moreno E, McCormick PJ, et al: The insulin-like growth factor I receptor regulates glucose transport by astrocytes. Glia 64 1962-1971, 2016

35. Attwell D and Laughlin SB: An energy budget for signaling in the grey matter of the brain. J Cereb Blood Flow Metab 21: $1133-1145,2001$

36. Wu J, Lin B, Liu W, Huang J, Shang G, Lin Y, Wang L, Chen L and Tao J: Roles of electro-acupuncture in glucose metabolism as assessed by 18 F-FDG/PET imaging and AMPK $\alpha$ phosphorylation in rats with ischemic stroke. Int J Mol Med 40: 875-882, 2017.

37. Simpson IA, Carruthers A and Vannucci SJ: Supply and demand in cerebral energy metabolism: The role of nutrient transporters. J Cereb Blood Flow Metab 27: 1766-1791, 2007.

38. Farrell CL and Pardridge WM: Blood-brain barrier glucose transporter is asymmetrically distributed on brain capillary endothelial lumenal and ablumenal membranes: An electron microscopic immunogold study. Proc Natl Acad Sci USA 88: 5779-5783, 1991

39. Duarte AI, Santos P, Oliveira CR, Santos MS and Rego AC: Insulin neuroprotection against oxidative stress is mediated by Akt and GSK-3beta signaling pathways and changes in protein expression. Biochim Biophys Acta 1783: 994-1002, 2008.
40. Hwang IK, Yoo KY, Park SK, An SJ, Lee JY, Choi SY, Kang JH, Kwon YG, Kang TC and Won MH: Expression and changes of endogenous insulin-like growth factor-1 in neurons and glia in the gerbil hippocampus and dentate gyrus after ischemic insult. Neurochem Int 45: 149-156, 2004.

41. Wadowska M, Woods J, Rogozinska M and Briones TL: Neuroprotective effects of enriched environment housing after transient global cerebral ischaemia are associated with the upregulation of insulin-like growth factor-1 signalling. Neuropathol Appl Neurobiol 41: 544-556, 2015.

42. Bergstedt $\mathrm{K}$ and Wieloch T: Changes in insulin-like growth factor 1 receptor density after transient cerebral ischemia in the rat. Lack of protection against ischemic brain damage following injection of insulin-like growth factor 1. J Cereb Blood Flow Metab 13: 895-898, 1993.

43. Cui H, Meng Y and Bulleit RF: Inhibition of glycogen synthase kinase 3beta activity regulates proliferation of cultured cerebellar granule cells. Brain Res Dev Brain Res 111: 177-188, 1998.

44. Johnston BM, Mallard EC, Williams CE and Gluckman PD Insulin-like growth factor-1 is a potent neuronal rescue agent after hypoxic-ischemic injury in fetal lambs. J Clin Invest 97: 300-308, 1996

45. Jiang LH, Yuan XL, Yang NY, Ren L, Zhao FM, Luo BX, Bian YY, Xu JY, Lu DX, Zheng YY, et al: Daucosterol protects neurons against oxygen-glucose deprivation/reperfusion-mediated injury by activating IGF1 signaling pathway. J Steroid Biochem Mol Biol 152: 45-52, 2015.

46. Liang K, Ye Y, Wang Y, Zhang J and Li C: Formononetin mediates neuroprotection against cerebral ischemia/reperfusion in rats via downregulation of the $\mathrm{Bax} / \mathrm{Bcl}-2$ ratio and upregulation PI3K/Akt signaling pathway. J Neurol Sci 344: 100-104, 2014.

47. Wu D, Shi J, Elmadhoun O, Duan Y, An H, Zhang J, He X, Meng R, Liu X, Ji X and Ding Y: Dihydrocapsaicin (DHC) enhances the hypothermia-induced neuroprotection following ischemic stroke via PI3K/Akt regulation in rat. Brain Res 1671: 18-25, 2017.

48. Yang L, Zhang Y, Yan Z and Tian F: The role of mTOR signaling pathway on cognitive functions in cerebral ischemia-reperfusion. Exp Ther Med 14: 2839-2844, 2017.

49. Li X, Ren C, Li S, Han R, Gao J, Huang Q, Jin K, Luo Y and Ji X: Limb remote ischemic conditioning promotes myelination by upregulating PTEN/Akt/mTOR signaling activities after chronic cerebral hypoperfusion. Aging Dis 8: 392-401, 2017.

50. Zhuang Q, Dai C, Yang L, Wen H, Wang H, Jiang X and Zhang Y: Stimulated CB1 cannabinoid receptor inducing ischemic tolerance and protecting neuron from cerebral ischemia. Cent Nerv Syst Agents Med Chem 17: 141-150, 2017

51. Gluckman P, Klempt N, Guan J, Mallard C, Sirimanne E, Dragunow M, Klempt M, Singh K, Williams C and Nikolics K: A role for IGF-1 in the rescue of CNS neurons following hypoxic-ischemic injury. Biochem Biophys Res Commun 182: 593-599, 1992

52. Wang JM, Hayashi T, Zhang WR, Sakai K, Shiro Y and Abe K: Reduction of ischemic brain injury by topical application of insulin-like growth factor-I after transient middle cerebral artery occlusion in rats. Brain Res 859: 381-385, 2000.

This work is licensed under a Creative Commons Attribution 4.0 International (CC BY 4.0) License. 